\title{
Outbreak of Hemolytic-Uremic Syndrome Linked to Shiga Toxin-Producing Enteroaggregative Escherichia coli O104:H4
}

$\mathbf{I}^{\mathrm{n}}$ nearly May 2011, authorities in northern Germany were alerted to an unusually high number of cases of bloody diarrhea and hemolytic uremic syndrome (HUS). Public health microbiologists observed that affected patients were not shedding the typical etiologic agent of this syndrome: Escherichia coli of serotype O157:H7, also known as enterohemorrhagic E. coli (EHEC). A thorough investigation implicated the real culprit: a rare E. coli serotype known as O104:H4. By the end of June, the outbreak peaked, with the European Union reporting 888 HUS cases, including 31 deaths, and 3189 non-HUS cases, including 17 deaths (1).

Noting precedent with EHEC and similar outbreaks, German epidemiologists scrambled to implicate a food source. Although initial investigations indicted cucumbers imported from Spain, the first questionnaires inadvertently omitted sprouts from the queried food items, thereby suggesting vegetables commonly served therewith. Revision of the questionnaires identified sprouts grown on a farm in Lower Saxony as the true villains, revealing the necessity of considering cotravelers in any epidemiologic investigation.

Remarkably, within $2 \mathrm{wk}$, at least three laboratory teams had sequenced the novel pathogen, depicting a fascinating tale of molecular evolution. Although the most virulent Shiga toxin-producing EHEC share common virulence features, notably the Shiga toxin itself and a chromosomal genetic island called the Locus of Enterocyte Effacement (LEE), the O104:H4 strain did not harbor the LEE or other elements characteristic of EHEC. Rather, the organism descended from a common but underappreciated $E$. coli pathogen called enteroaggregative E. coli (EAEC).

EAEC may be the most common bacterial cause of diarrhea in the United States (2) and perhaps in other parts of the world as well. It causes diarrhea by adhering abundantly to the small and large intestinal mucosal surfaces by virtue of Aggregative Adherence Fimbriae. Once colonized, the bacteria secrete enterotoxins and cytotoxins, the combined effect of which is to induce tissue destruction, opening of tight junction barriers, and establishment of a net secretory state. Interestingly, not all strains that fit the EAEC definition (characteristic aggregating adherence to eukaryotic cells) are genuine human pathogens; rather, strains must harbor a complete set of virulence factors, which may be inherited piecemeal. The O104:H4 may provide insights into the essential EAEC virulence determinants.
Molecular sleuths have unraveled an unexpected story behind the outbreak strain. O104:H4 E. coli apparently circulates in Africa, where it carries the plasmid and chromosomal virulence determinants characteristic of EAEC. This author's observations suggest that this serotype may be more commonly found in individuals with diarrhea, although formal clinical and epidemiologic data to this effect are not available. Interestingly, other strains of EAEC have become lysogenized with the Shiga toxin-encoding phage, and two such O104:H4 strains were isolated from the Republic of Georgia (although not from outbreaks). The German outbreak strain represents the climax of the clone's evolutionary ascent, because the organism features not only the Shiga toxin but also harbors multiple antibiotic resistance genes.

Moreover, the outbreak highlights the remarkable genomic plasticity of E. coli as a bacterial species. Considering all E. coli genomes sequenced to date reveals that only about 2200 genes $(\sim 50 \%)$ of the open reading frames in any particular isolate are conserved among all strains (the "core"); these genes serve essential functions of metabolism and fission. The remaining genes are shuttled about by various mechanisms of horizontal exchange, principally plasmid conjugation and phage transduction. Although the ability to exchange genes predicts an amorphous phylogenetic structure, there is general conservation of genes and mechanisms within each enteric pathotype (enterotoxigenic, enteropathogenic, enteroaggregative, enteroinvasive, and enterohemorrhagic/Shiga toxin-producing), suggesting an element of evolutionary purification.

The most compelling question raised by this outbreak surrounds the future of the outbreak strain. EAEC are endemic in nearly all human populations (although EHEC are not), but such endemic strains do not secrete Shiga toxin. The unusual O104:H4 pathotype chimera may be unfit for epidemiologic survival, or it may achieve ecological success and find a new niche in human or animal populations. In either case, we mark a new chapter in the history of an extraordinary microbial species.—James P. Nataro

\section{REFERENCES}

1. European Centre for Disease Prevention and Control 2011 ECDC update on outbreak in Germany and cluster in France. Available at: ecdc.europa.eu/en/activities/sciadvice/. Accessed June 27, 2011

2. Nataro JP, Mai V, Johnson J, Blackwelder WC, Heimer R, Tirrell S, Edberg SC, Braden CR, Glenn Morris J Jr, Hirshon JM 2006 Diarrheagenic Escherichia coli infection in Baltimore, Maryland, and New Haven, Connecticut. Clin Infect Dis 43:402-407 